\title{
A framework for the optimal design of a minimum set of clinical trials to characterize von Willebrand disease
}

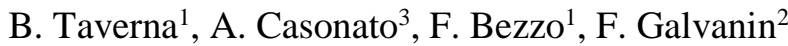

${ }^{1}$ CAPE-Lab (Computer-Aided Process Engineering Laboratory), Department of Industrial Engineering, University of Padua

${ }^{2}$ Department of Chemical Engineering, University College London,

${ }^{3}$ Department of Medicine, University of Padua Medical School 


\section{Abstract}

Background and Objective: Von Willebrand disease (VWD) is one of the most severe inherited bleeding disorder in humans, and it is associated with a qualitative and/or quantitative deficiency of von Willebrand factor, a multimeric glycoprotein fundamental in the coagulation process. At present, the diagnosis of VWD is extremely challenging and mostly based on clinical experience. Kinetic models have been recently proposed and applied to help in the diagnosis and characterization of VWD, but the complexity of these models is such that they requires long and stressful clinical tests, such as the desmopressin response test (DDAVP), to achieve a satisfactory estimation of the individual haemostatic parameters. The goal of this paper is to design a minimal set of clinical tests for the identification of akinetic model to decrease the required time and effort for the characterization and diagnosis of VWD.

Methods: A model proposed in the literature is used as a building block to develop a new model, where response surface methodologies have been applied to determine a set of explicit correlations linkingkinetic model parameters to basal clinical trials data. Model-based design of experiments techniques are then used to devise optimally informative tests for model validation which are shorter and easier to implement.

Results: Results show an excellent agreement between the original model for VWD and the new proposed model on representing healthy and VWD subjects. The application of experimental design techniques for model validation shows the possibility to drastically reduce the duration of DDAVP tests from $24 \mathrm{~h}$ to $3 \mathrm{~h}$ by exploiting complementary information from basal clinical tests.

Conclusions: Basal clinical tests can be used alongside a time-reduced DDAVP test to validate pharmacokinetic models for a quantitative characterisation of subjects affected by VWD and for a quicker and easier diagnosis of the disease.

Keywords: von Willebrand disease; pharmacokinetics; optimal design of clinical tests; pharmacokinetic models; basal clinical tests 


\section{Introduction}

Von Willebrand disease (VWD) is one of the most diffuse bleeding disorders visible in humans, caused by a dysfunction of von Willebrand factor (VWF), a multimeric glycoprotein present in the bloodstream [17]. VWF acts as a fundamental vector in the haemostatic process by mediating platelet aggregation and thrombus growth and by binding, transporting and protecting coagulation factor VIII. VWD can be defined as an alteration of VWF in the bloodstream with symptoms ranging from sporadic nosebleeds and mild bleeding from scars or small lesions in skin to acute thrombocytopenia and/or protracted bleeding events [21]. A clear diagnosis of VWD may be difficult due to the existence of several VWD types (1, 2A, 2B, Vicenza, 2M, 2N, 3) [14,24]. Kinetic models have been recently developed for the classification of the disease, elucidating the critical pathways involved in the disease characterization and paving the way to new approaches for model-based diagnosis of VWD $[8,11,13]$. However, the degree of complexity of these models is such that they require the execution of extremely time-consuming tests (lasting at least $24 \mathrm{~h}$ ) like the desmopressin response test (DDAVP) to be carried out on the subjects to estimate the individual haemostatic parameters. During a DDAVP test desmopressin is administered subcutaneously at a dose of $0.3 \mathrm{~g} / \mathrm{kg}$ to patients, and blood samples are collected at regular times $(15,30,60,120,180$, 240, $480 \mathrm{~min}$ and $24 \mathrm{~h}$ after the injection). DDAVP induces an acute release of VWF stored in the Weibel Palade bodies of the endothelial cells, so the time course of VWF antigen (VWF:Ag) and VWF collagen binding (VWF:CB) after DDAVP can be quantitatively analysed by mean of models describing the kinetics of variation in concentration $[7,18]$. The levels of VWF in plasma depend on three determinants: $i$ ) the amount and rate of VWF release; ii) ADAMTS-13 proteolytic activity; and iii) VWF clearance from plasma. The main issues linked with the use of DDAVP as mean of detection are the fact that this test is a time-consuming and cumbersome non-routine test, which needs to be carried out in specialized facilities to achieve a statistically satisfactory estimation of the individual haemostatic parameters. Therefore, the scientific community is studying a way for identifying the kinetic model of VWD without the need of the DDAVP test, exploiting only basal 
clinical tests. Beside the evaluation of VWF:Ag and VWF:CB at the basal state, alternative promising tests have been recently considered: $i$ ) the activated conformational state test [14], used to evaluate VWF functional activity; ii) the propeptide test (VWFpp) [6] used to quantify the VWF elimination from the blood stream; iii) the interplatelet von Willebrand factor [22] to quantify the amount of VWF synthesized in the endothelial cells.

The estimation of kinetic parameters in physiological systems is usually carried out using nonlinear parameter estimation techniques [4] applied to a population of subjects $[2,9,11]$. However, a comprehensive and reliable definition of the variability of kinetic parameters for a population usually requires an expensive, lengthy and time-consuming procedure, particularly for rare diseases such as VWD. Techniques have been recently proposed in the process systems engineering community to guarantee a reliable estimation of parameters in kinetic models from limited data sets [15]. However, so far no attempt was carried out for the design of alternative clinical tests for the identification of kinetic models of VWD, to reduce time and cost of clinical experimentation.

In this paper a minimal set of clinical trials, including both basal tests (VWF:Ag, VWF:CB and VWFpp) and an optimised dynamic test (DDAVP), is proposed for the identification of kinetic models of VWD. A new kinetic model, based on modifications of the VWD model proposed by Ferrari and coworkers [11], has been developed to include in the formulation a set of explicit correlations linking the model parameters to basal clinical trials measurements using response surface methodology (RSM). The new model was then used to optimise the clinical protocols using model-based design of experiments (MBDoE) techniques [3,10,12]. These techniques aim at maximizing the information content of the experimental measurements by optimising the allocation of sample measurements in time [10,23], thus minimizing the expected parameter variances, which are then related to the uncertainty on the estimated kinetic parameters [4]. Results show how a minimal set of clinical tests can be used where a reduced DDAVP test (lasting 3 hours) is employed alongside basal clinical trials (VWF:Ag, VWF:CB and VWFpp tests) for the quick identification of 
the kinetic model of VWD in order to decrease the time and effort required for VWD characterization and diagnosis.

\section{Methods}

Model modification has been carried out using clinical data derived by 20 VWD patients belonging to $2 \mathrm{~B}$ and Vicenza categories and 42 normal subjects with $\mathrm{HnonO}$ and $\mathrm{HO}$ blood group. Patients and normal subjects were studied in accordance with the Helsinki Declaration, after obtaining their written informed consent, and our ethical board's approval of the study. Clinical data have been supplied by the Hospital of Padua.

Diagnostic procedures for VWD may entail the use of both basal (time-independent) clinical tests and dynamic (time-dependent) tests, which are detailed in the following subsections.

\subsection{Clinical tests and data: basal clinical tests}

\subsubsection{VWF antigen (VWF:Ag)}

VWF:Ag, to evaluate the concentration of VWF molecule in the bloodstream, was measured by a home-made ELISA method. Platelet VWF:Ag, to evaluate the synthesis of VWF, was measured with a home-made ELISA method, using washed platelets, adjusted to 1 million and lysed with Triton X-100. A pool of normal washed platelets was used to construct the reference curve. The results are given in U/dL, taking the first reference curve dilution as $100 \mathrm{U} / \mathrm{dL}$.

\subsubsection{VWF collagen binding (VWF:CB)}

VWF:CB, to evaluate the ability of VWF in binding with collagen, was assessed by an ELISA method using type III collagen (Sigma, Milan, Italy) diluted in acetic acid. The results are given in $\mathrm{U} / \mathrm{dL}$, taking the first reference curve dilution as $100 \mathrm{U} / \mathrm{dL}$.

\subsubsection{VWF propeptide (VWFpp)}

VWFpp, to evaluate the elimination of VWF from the bloodstream, was measured using a homemade ELISA method. Briefly, diluted reference and patient plasma samples were added to 
microwells on microtitration plates coated with a monoclonal antibody specific for VWFpp (CLBPro 35, Sanguin, The Netherlands); and bound VWFpp was assessed with a second anti-VWFpp HRP-labelled monoclonal antibody (M193904, Sanguin). The results are given in U/dL, taking the first reference curve dilution as $100 \mathrm{U} / \mathrm{dL}$.

\subsubsection{VWF:CB ratio and VWFpp ratio}

VWFpp, whose evaluation is used to assess the survival of VWF, is secreted by endothelial cells as a dimer, in a ratio of 1:1 with mature VWF. While mature polymerized VWF survives for around 10-20 hours, VWFpp has a half-life of just 1-2 hours. No pathological conditions or mutations are known to affect the survival of VWFpp, while a number of VWF mutations are known to affect the half-life of mature VWF. That is why the VWFpp/VWF:Ag ratio (VWFpp ratio) gives an indirect measure of VWF survival. A reduced VWF half-life coincides with an increase in the VWFpp ratio: the higher the VWFpp ratio, the shorter the survival of VWF.

VWF:CB measures the capacity of VWF to bind to extravascular collagen. This binding relies on the integrity of the collagen binding domain of VWF (A1 and A3 domains), as well as the presence of large VWF multimers (the multimeric components being the more efficient in binding collagen). A lower VWF:CB/VWF:Ag ratio (VWF:CB ratio) is suggestive of a reduction in, or disappearance, of large VWF multimers or, less frequently, of altered collagen binding domains of VWF. A greater reduction in the VWF:CB ratio coincides with a more pronounced shortage of large VWF multimers.

\subsection{Clinical tests and data: dynamic tests}

\subsubsection{DDAVP}

DDAVP (1-desamino-8-D-argine vasopressin; Emosint, Sclavo, Italy) was administered subcutaneously at a dose of $0.3 \mu \mathrm{g} \mathrm{kg}^{-1}$. Blood samples were collected before and 15, 30, 60, 120, 180, 240, 480 min and $24 \mathrm{~h}$ after administering DDAVP. An example of DDAVP time course is given in Figure 1 for representative subjects from each category: healthy subjects $(\mathrm{O} /$ non-O blood group) and subjects affected by VWD type 2B and Vicenza. 


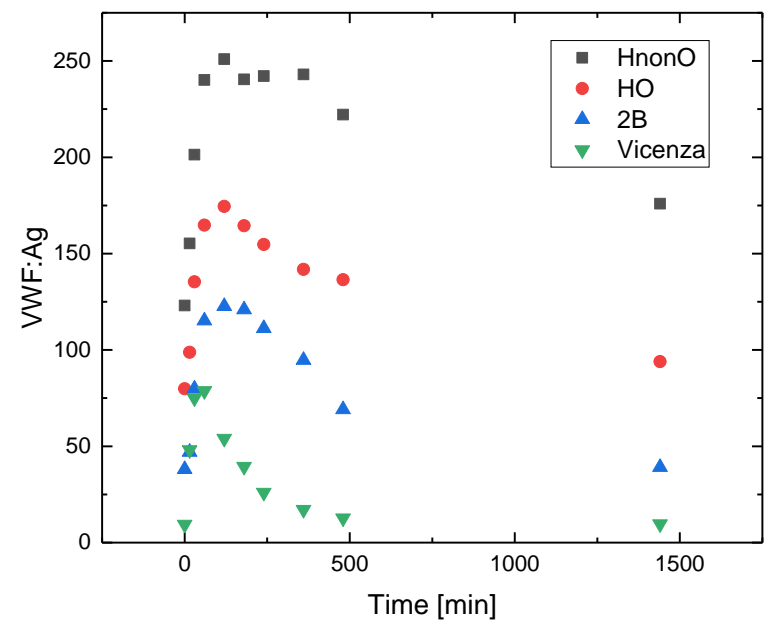

(a)

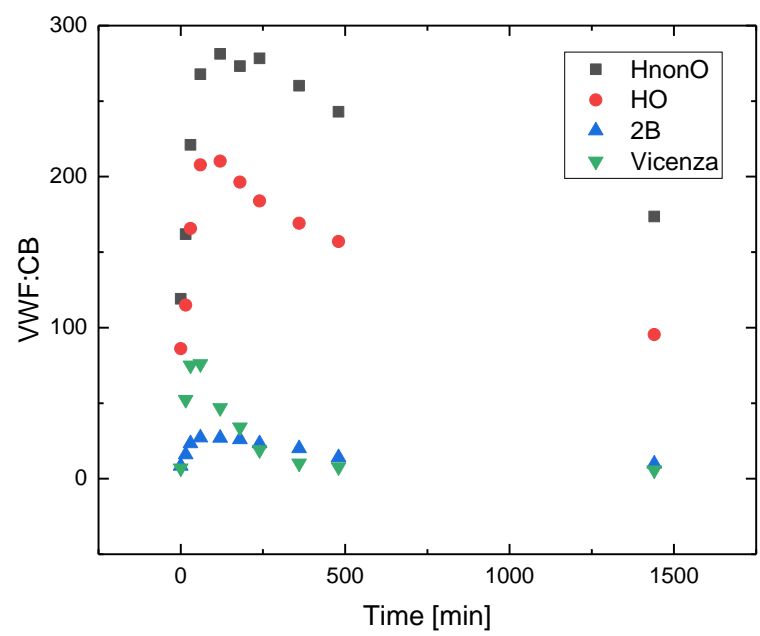

(b)

Figure 1. Examples of (a) VWF:Ag and (b) VWF:CB measurements after DDAVP execution for the classes of subjects analysed in the study (healthy O/non-O subjects, subjects affected by VWD type Vicenza and $2 B$ ).

The time course of plasma VWF:Ag and VWF:CB levels after the DDAVP can be analyzed to obtain important information on the three basic pathways defining VWF distribution in the blood stream: VWF release, VWF proteolysis and VWF elimination.

\subsection{Proposed methodology}

The proposed procedure to design a minimum set of clinical tests for the diagnosis of VWD is summarized in the block-diagram reported in Figure 2. In the first step the model proposed in [11] is used to identify the haemostatic parameters for each relevant class of subjects $(2 \mathrm{~A}, 2 \mathrm{~B}$, Vicenza, Healthy O, Healthy Non-O) from DDAVP data. In the second step the information from basal clinical data (VWF:Ag, VWF:CB and VWF:pp) is used to build regression models for the elimination rate constant (first level of model modification) and proteolysis rate constant (second level of model modification). In the third step the RSM models determined in step 2 are used to define a new modified model including the information from basal clinical trials. Finally (step 4) the modified model is used to optimally design a minimum set of clinical tests for achieving a statistically reliable estimation of kinetic parameters, and thus a precise hemostatic portrait of each single individual to support model-based diagnosis. 


\subsubsection{Available clinical dataset}

Clinical data from the basal and dynamic test illustrated in Section II are available for each pool of subjects. The features of the available dataset are illustrated in Table 1.

\subsubsection{Identification of the PK model of VWD}

The measurements derived from the DDAVP test and reported in Supplementary Material 3 were used to obtain a first estimation of the parameters of the model of VWD proposed in [11]. The model assumes that after DDAVP administration, both high molecular weight (HMW) and ultralarge molecular weight (UL) VWF multimers are released from the endothelial cells. The HMW and UL multimers are then cleaved to low molecular weight (LMW) multimers by the metalloprotease ADAMTS-13 and finally eliminated from the bloodstream. The model is described by a system of differential and algebraic equations where each subject is characterized using three main kinetic constants, namely the VWF release rate $k_{0}\left[\mathrm{~h}^{-1}\right]$, the proteolysis rate $k_{1}\left[\mathrm{~h}^{-1}\right]$ and the elimination rate $k_{\mathrm{e}}\left[\mathrm{h}^{-1}\right]$, which is assumed to be independent from multimers size [16]. Details on the kinetic model and parameter estimation procedure can be found in Supplementary Material 1. 


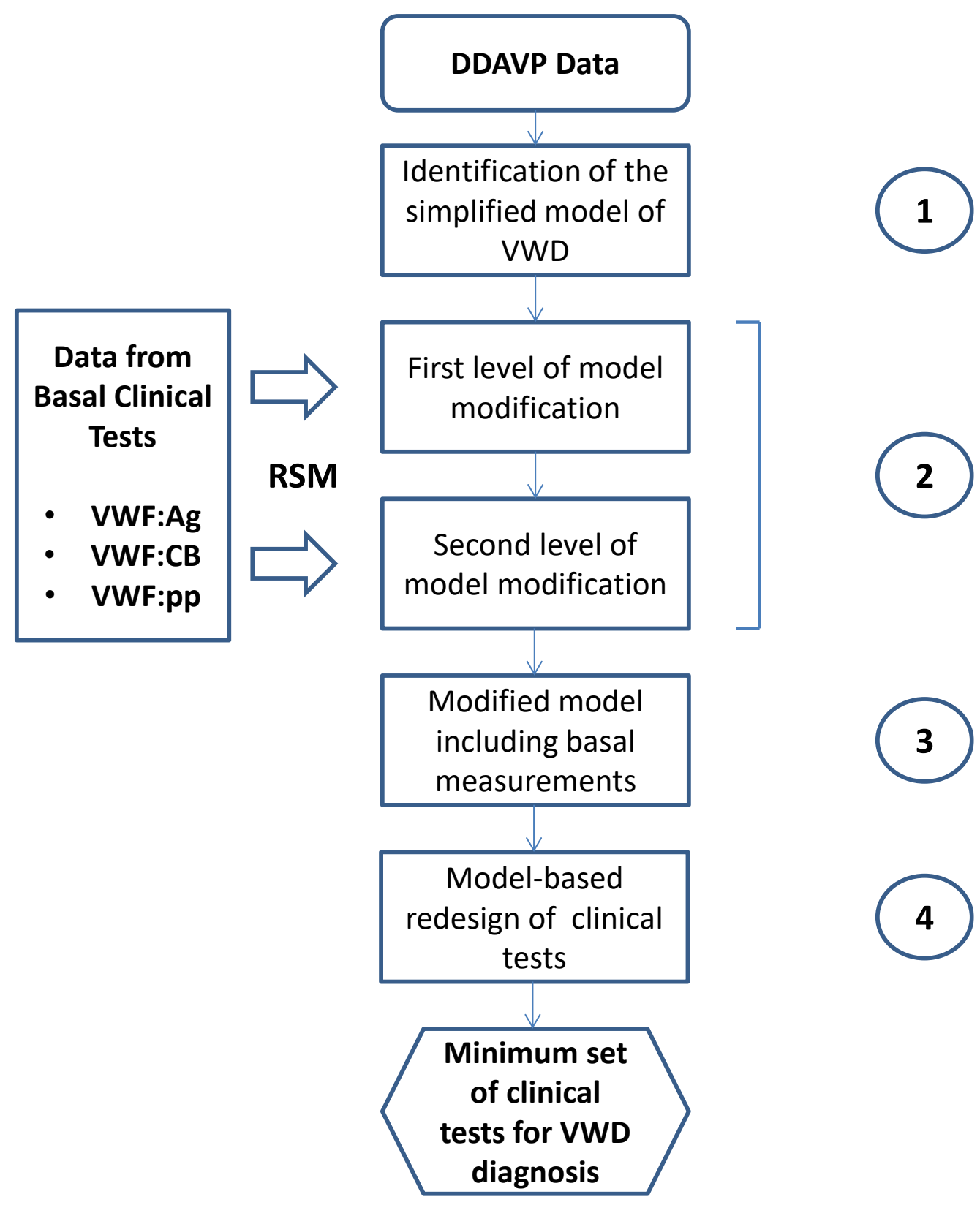

Figure 2. Proposed modelling procedure.

Table 1. Illustration of the features of the available dataset.

\begin{tabular}{|c|c|c|c|c|c|c|}
\hline Subjects & $\begin{array}{c}\text { Number of } \\
\text { subjects }\end{array}$ & $\begin{array}{c}\text { Age } \\
\text { years }\end{array}$ & $\begin{array}{c}\text { Body Weight } \\
\text { Kg }\end{array}$ & $\begin{array}{c}\text { Blood group } \\
\text { O/nonO }\end{array}$ & $\begin{array}{c}\text { VWF:Ag } \\
\text { U/dL }\end{array}$ & $\begin{array}{c}\text { VWF:CB } \\
\text { U/dL }\end{array}$ \\
\hline Vicenza & 9 & $26-59$ & $67-106$ & $2 / 7$ & $8.8 \pm 2.2$ & $7.2 \pm 2.6$ \\
\hline 2B & 8 & $36-65$ & $50-91$ & $5 / 3$ & $41.3 \pm 9.5$ & $8.9 \pm 4.2$ \\
\hline Normal subjects & 42 & $19-52$ & $43-95$ & $17 / 25$ & $96.3 \pm 46.5$ & $99.4 \pm 45.9$ \\
\hline Normal range & - & - & - & - & $60-160$ & $65-150$ \\
\hline
\end{tabular}




\subsubsection{First and second level of model modification using response surface methodologies}

The model [11] is affected by two important limitations: $i$ ) parameters need to be estimated from the DDAVP test; $i$ ) it does not consider the availability of basal clinical trials such as VWF:pp (which can be useful to quantify VWF elimination) in the equations set. Basal clinical data (as described in section 2) and the estimates of model parameters from DDAVP data are available from DDAVP for each patient (details are reported in Supplementary Material 2). These data have been used for the development of correlations using response surface methodology (RSM) which relate the model parameters $k_{e}$ and $k_{1}$ estimated from DDAVP to measurements acquired from basal clinical trials (VWF:pp and VWF:CB ratio used to quantify, respectively, VWF elimination and relative deficiency of high molecular weight VWF multimers). RSM is a technique originally proposed by Box-Behnken [5], used to define "black-box" models which allow to investigate possible correlations between inputs and outputs in systems where the intrinsic behavior is either physically unknown or difficult to represent. Basically, the objective consists into hypothesizing an analytical form of the response surface fitting the experimental data. Mathematically, the response-surface model is a $k$-dimensional hyper-surface acting in a space which is $(k+1)$ dimensional. In this case, a suitable correlation has to be found for one single parameter that depends on measurements from two basal clinical trials. Therefore, the workspace is $3 \mathrm{D}$, whereas the hypersurface is $2 \mathrm{D}$. The generic RSM model takes the following form

$$
Y=Y_{0}+\sum_{i=1}^{N_{F}} \alpha_{i} F_{i}+\sum_{i=1}^{N_{F}} \beta_{i} F_{i}^{2}+\sum_{(i \neq j)=1}^{N_{F}} \gamma_{i} F_{i} F_{j}+\sum_{(i \neq j)=1}^{N_{F}} \delta_{i}\left(F_{i} F_{j}\right)^{2} \quad i=1 \ldots N_{F}
$$

where $Y$ is the observed response (in this case the kinetic parameter), $F_{i}$ represents the $i$-th experimental observation from basal clinical data, $N_{F}$ is the number of factors obtained from basal clinical data and $Y_{0}, \alpha_{i}, \beta_{i}, \gamma_{i}, \delta_{i}$ are the model parameters to be estimated.

The most suitable model is selected based on a criterion of parsimony where, together with the model (fitting) performance, also model complexity (number of parameters to be estimated) needs 
to be considered. The Akaike information criterion (AIC) [1] has been used at this purpose as a measure of the relative quality and complexity of statistical models. Given a set of regression models and a set of clinical data, AIC provides a tool for model selection. The AIC for a model is mathematically expressed as:

$$
\mathrm{AIC}=2 k-2 \ln (L)
$$

where $k$ is the number of model parameters and $L$ is the maximum value of the likelihood function for the model. In the fitting procedure, the values of the parameters are evaluated through the Least Squared Method (LSM), suitable for overdetermined systems. Data analysis and response surface modelling have been carried out in OriginPro ${ }^{\circledR}$ [19]. After data fitting, it is important to statistically evaluate the goodness of fit. This has been evaluated using the following metrics [4]:

- Residuals sum of squares : to show the total deviation of the response values from the fit to the response values;

- $\quad$ R-square $\left(R^{2}\right)$ statistic: to measure how successful the fit is in explaining the variation of the data $\left(R^{2}\right.$ can take only values between 0 and 1 , a value closer to 1 indicates a better fit);

- $\bar{R}^{2}$ statistic: if the number of fitted coefficients in the model increases, $R^{2}$ might increase although the fit may not improve. To avoid this situation, the $\bar{R}^{2}$ statistic can be used as it takes into account the degrees of freedom of the estimates and of the system considered. The $\bar{R}^{2}$ statistic can only have values less or equal to 1 , with a value closer to 1 indicating a satisfactory fit.

\subsubsection{Modified model including basal measurements}

The RSM correlations discussed in the previous section have been substituted in the original model. This procedure leads to a first and to a second level of model modification. In the first level of model modification the RSM correlation in the form given by (1) is provided for parameter $k_{e}$ (VWF elimination rate constant); in the second level of model modification the RSM correlation is provided for parameter $k_{1}$ (VWF proteolysis rate constant). The modified model of VWD has been 
then validated by simulation of the VWF:Ag and VWF:CB profiles of response of four subjects that do not belong to the original pool of subjects (validation set) and the profiles have been compared to the ones obtained with the original model of VWD.

\subsubsection{Model-based redesign of clinical tests}

Information content analysis has been carried out on both the original model [11] and on the proposed new model of VWD with the following goals: $i$ ) understanding the DDAVP execution time which is required to estimate the kinetic parameters; ii) evaluating the relative impact of complementary information provided by basal clinical tests on parameter estimation; iii) provide the optimal allocation of sampling points, known as optimal sampling scheduling (OSS) [23]. The metric used to evaluate the information content of a clinical trial is the trace of Fisher Information Matrix (FIM) [4], which is defined by

$$
I_{d}=\sum_{n_{s p}} \operatorname{tr}\left[\mathbf{H}_{\theta}\right]=\sum_{n_{s p}} \operatorname{tr}\left\{\sum_{i=1}^{N_{e x p}} \sum_{j=1}^{N_{m}}\left[\frac{1}{\sigma_{i j}^{2}}\left(\frac{\partial \hat{y}_{i j}}{\partial \theta_{k}} \frac{\partial \hat{y}_{i j}}{\partial \theta_{l}}\right)\right]_{k, l=1 \ldots N_{\theta}}\right\}
$$

In (3) $n_{s p}$ is the total number of sampling points and $\mathbf{H}_{\theta}$ is the FIM related to each single sampling point obtained from $N_{\exp }$ clinical tests (basal or dynamic). The FIM, which is the inverse of the variance-covariance matrix of model parameters, is expressed as the product of the sensitivity of the $j$-th output variable with respect to each of the $N_{\theta}$ parameter in the conditions investigated in the $i$-th experiment, divided for the corresponding variance of the experimental measurements for the $j$-th measured response in the $i$-th test $\left(\sigma_{i j}^{2}\right)$. An example of analysis of the time-dependent information profile (trace of FIM) is given in Figure 3. The maximum in the trace of FIM given by (3) defines the OSS to obtain the most informative samples from the clinical test. When the maximum of the information is located at the end of the test and the information is low at the beginning (Scenario 1, dashed line) all the samples should be concentrated at the very end of the trial (empty circles). In this case the information acquisition is favored by long test durations. If an information peak is located at the beginning (Scenario 2, solid line), the test can be usefully shortened as the samples can be concentrated in the first few hours of test execution (solid grey squares). 


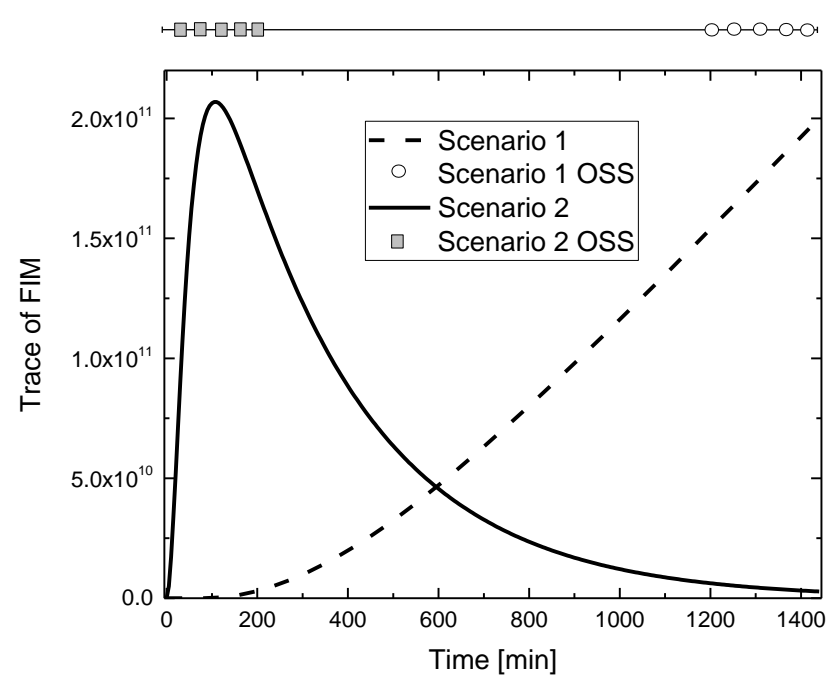

Figure 3. Analysis of information profiles: optimal sampling schedule (OSS) for different information scenarios.

\section{Results and discussion}

\subsection{Identification of a new kinetic model including basal measurements}

Basal (VWF:Ag, VWF:CB, VWFpp) and dynamic (DDAVP) clinical data were available for each subject considered in the pool. The subject-specific DDAVP data have been used to estimate the model kinetic parameters $\left(k_{0}, k_{1}, k_{\mathrm{e}}, D\right.$ and $\left.t_{\max }\right)$ for each subject. The values of the model parameters together with the basal clinical data represent the experimental dataset (see Supplementary Material 2 for further details) through which suitable basal correlations have been investigated using RSM. Model discrimination results in terms of AIC index for linear and quadratic response surface models including interactions are reported in Table 2. The typology of response surface that has been found suitable is the linear response surface model with interactions. For this model the AIC is lower compared with that calculated using a quadratic model for all the considered categories. 
Table 2. AIC index for linear and quadratic response surface with interactions.

\begin{tabular}{ccc}
\hline & \multicolumn{2}{c}{ AIC INDEX } \\
\hline \hline & Linear response surface & Quadratic response surface \\
HnonO & $4.64 \mathrm{E}+01$ & $4.74 \mathrm{E}+01$ \\
$\mathrm{HO}$ & $4.10 \mathrm{E}+01$ & $4.44 \mathrm{E}+01$ \\
$2 \mathrm{~B}$ & $4.06 \mathrm{E}+01$ & $4.21 \mathrm{E}+01$ \\
Vicenza & $3.33 \mathrm{E}+01$ & $4.44 \mathrm{E}+01$ \\
\hline
\end{tabular}

Two basal state correlations have been defined, which allow to calculate, respectively, the elimination kinetic constant $k_{\mathrm{e}}$ and the proteolytic kinetic constant $k_{1}$ from basal clinical trials (VWF:Ag, VWF:CB and VWFpp) and the related basal quantities VWFpp ratio and VWF:CB ratio (indicated in the equations as VWF:R):

$k_{\mathrm{e}}=A 1+B 1 \cdot$ VWFpp ratio $+C 1 \cdot \mathrm{VWF}: \mathrm{R}+D 1 \cdot \mathrm{VWFpp}$ ratio $\cdot$ VWF: $\mathrm{R}$

$k_{1}=A 2+B 2 \cdot \mathrm{VWF}: \mathrm{Ag}+C 2 \cdot \mathrm{VWFpp}$ ratio $+D 2 \cdot \mathrm{VWF}: \mathrm{Ag} \cdot \mathrm{VWFpp}$ ratio

Considering at first the correlation for the elimination kinetic constant $k_{\mathrm{e}}$, the relation introduces 4 new model parameters (A1, B1, C1, D1) which have been directly obtained from the fitting procedure executed in OriginPro ${ }^{\circledR}$. Parameter estimation results and fitting statistics are given in Table 3, while surface fitting is visualized in Figure 4. As visible in Figure 4, the fitting appears to be good for all the categories as confirmed by the goodness of fit statistics reported in Table 3 , which shows a $R^{2}$ higher than $90 \%$ in all the subjects categories. The new explicit correlation (4) has been substituted into the kinetic model of VWD leading to a first level of model modification. Results from Table 4 show that the correlation allows a correct calculation of $k_{\mathrm{e}}$, as the relative error based on the difference between $k_{\mathrm{e}}$ calculated with the first level modified model and estimated with the original model is always below $10 \%$. 
Table 3. Estimated values for the parameters of the RSM model used for calculating the elimination kinetic constant $k_{e}$ and goodness of fit statistics.

\begin{tabular}{|c|c|c|c|c|c|c|c|c|}
\hline \multirow{2}{*}{$\begin{array}{c}\text { Subject } \\
\text { Category }\end{array}$} & \multicolumn{4}{|c|}{ Estimated values } & \multicolumn{4}{|c|}{ Goodness of fit statistics } \\
\cline { 2 - 9 } & A1 & B1 & C1 & D1 & DoF & Residuals sum of squares & $\boldsymbol{R}^{2}$ & $\overline{\boldsymbol{R}}^{2}$ \\
\hline HnonO & 0.01468 & -0.01521 & -0.01316 & 0.01438 & 4 & $8.09 \mathrm{E}-08$ & 0.926 & 0.750 \\
\hline HO & -0.01200 & 0.01311 & 0.01089 & -0.01030 & 5 & $4.62 \mathrm{E}-07$ & 0.910 & 0.724 \\
\hline 2B & 0.00930 & -0.00348 & -0.02435 & 0.01299 & 2 & $2.69 \mathrm{E}-07$ & 0.979 & 0.896 \\
\hline Vicenza & 0.02005 & -0.00162 & 0.00916 & 0.00138 & 1 & $3.19 \mathrm{E}-07$ & 0.929 & 0.455 \\
\hline
\end{tabular}

Table 4. Calculated average $k_{e}$ values and the average estimated $k_{e}$ values.

\begin{tabular}{cccc}
\cline { 2 - 4 } & $\begin{array}{c}k_{\mathrm{e}} \text { calculated by the first level } \\
\text { modified model }\end{array}$ & $\begin{array}{c}k_{\mathrm{e}} \text { estimated through the } \\
\text { original model }\end{array}$ & $\begin{array}{c}\text { relative } \\
\text { error }\end{array}$ \\
\cline { 2 - 4 } HnonO & $6.73 \mathrm{E}-04$ & $7.04 \mathrm{E}-04$ & 0.046 \\
$\mathrm{HO}$ & 0.00186 & 0.00152 & 0.082 \\
2B & 0.00333 & 0.00323 & 0.031 \\
Vicenza & 0.00841 & 0.00818 & 0.027
\end{tabular}

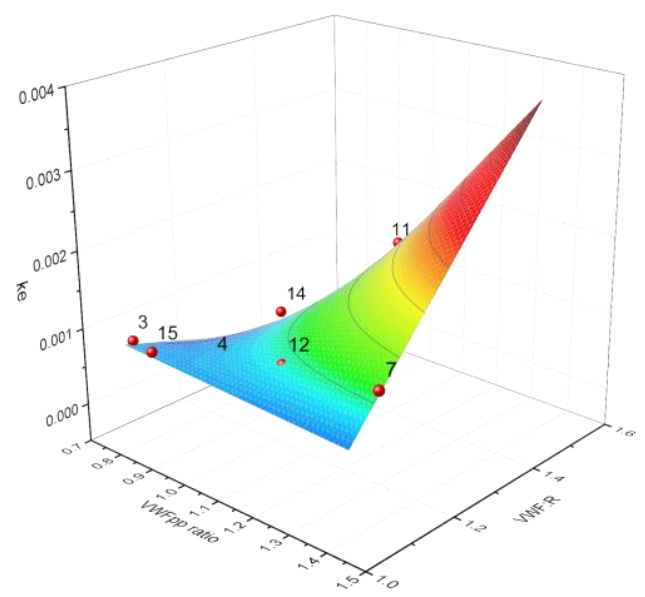

(a)

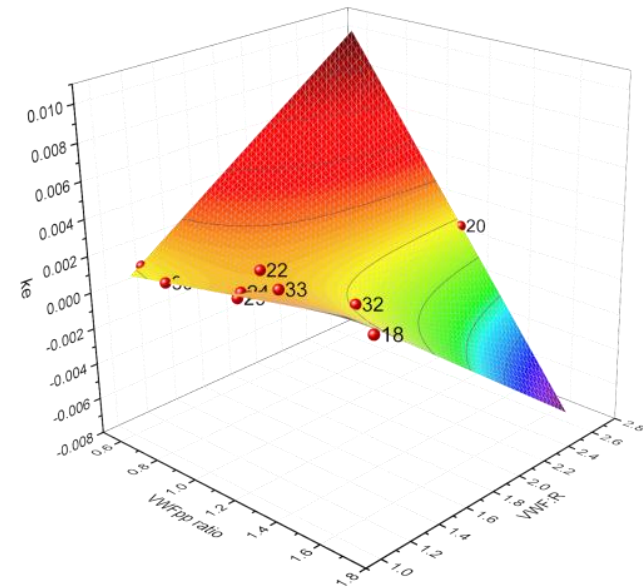

(b) 


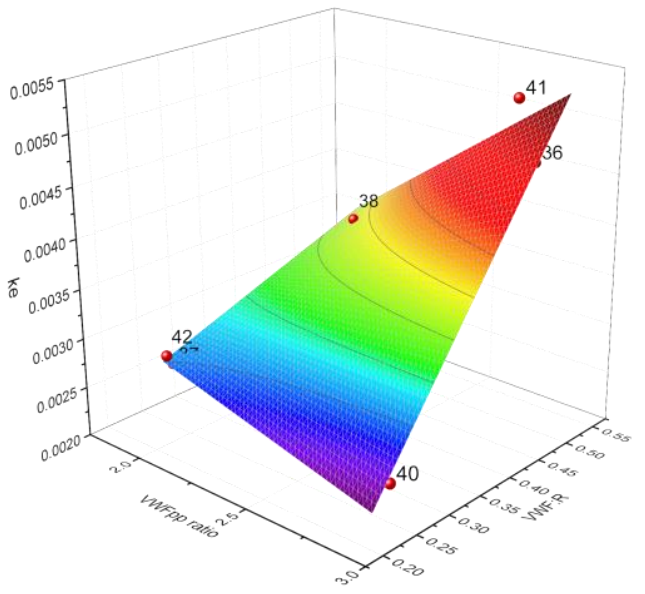

(c)

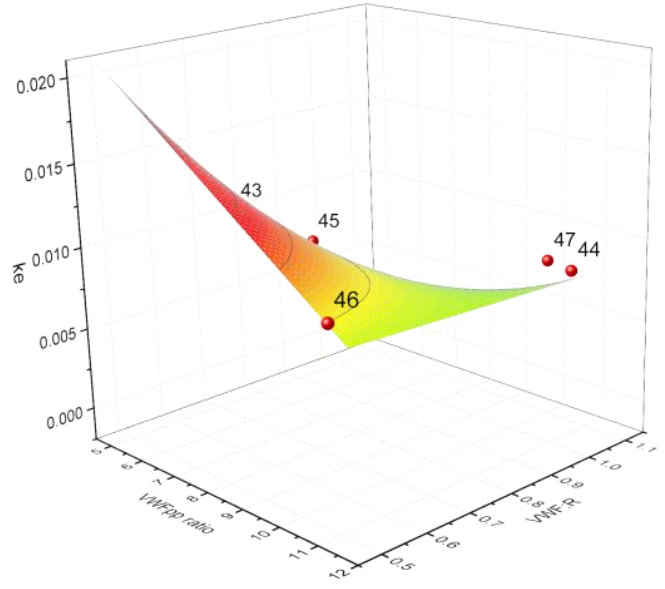

(d)

Figure 4. Linear response model surface with interaction for the elimination kinetic constant $k_{e}$ considering subjects HnonO (a), HO (b), 2B (c), Vicenza $(d)$.

Considering the correlation (5) for the proteolytic kinetic constant $k_{1}$ parameter estimation results and fitting statistics are given in Table 5, while surface fitting performance is shown in Figure 5. From the figure the fitting appears to be good for all the categories of subjects. However, as shown by the goodness of fit statistics reported in Table 5 , a $R^{2}$ higher than $90 \%$ is realized for HnonO, 2B and Vicenza type subjects, while the HO category shows a stronger heterogeneity on the estimated values of proteolytic constant $\left(R^{2}=0.725\right)$. The correlation is able to correctly calculate the proteolytic kinetic constant $k_{1}$ for all the categories and the relative error based on the difference between $k_{1}$ calculated with the second level modified model and estimated with the original model is lower than $10 \%$ (Table 6) except for HO subjects, where the deviation (27\%) is affected by the strong variability of proteolytic constants observed in this group.

Table 5. Estimated values for the parameters of the RSM model used for calculating the proteolytic kinetic constant $k_{l}$ and goodness of fit statistics.

\begin{tabular}{|c|c|c|c|c|c|c|c|c|}
\hline \multirow{2}{*}{$\begin{array}{c}\text { Subject } \\
\text { Category }\end{array}$} & \multicolumn{4}{|c|}{ Estimated values } & \multicolumn{4}{c|}{ Goodness of fit statistics } \\
\cline { 2 - 9 } & A2 & B2 & C2 & D2 & DoF & Residuals sum of squares & $\boldsymbol{R}^{\mathbf{2}}$ & $\overline{\boldsymbol{R}}^{\mathbf{2}}$ \\
\hline HnonO & -0.00312 & $4.09 \mathrm{E}-05$ & 0.00233 & $-2.96 \mathrm{E}-05$ & 4 & $4.43 \mathrm{E}-08$ & 0.917 & 0.722 \\
\hline $\mathrm{HO}$ & $-7.32 \mathrm{E}-04$ & $2.75 \mathrm{E}-05$ & 0.00174 & $-3.60 \mathrm{E}-05$ & 12 & $1.18 \mathrm{E}-06$ & 0.725 & 0.408 \\
\hline 2B & -0.01163 & $4.25 \mathrm{E}-04$ & 0.00432 & $-1.28 \mathrm{E}-04$ & 1 & $8.25 \mathrm{E}-07$ & 0.937 & 0.510 \\
\hline Vicenza & -0.00368 & $8.15 \mathrm{E}-04$ & $1.36 \mathrm{E}-04$ & $-5.04 \mathrm{E}-05$ & 1 & $7.36 \mathrm{E}-09$ & 0.999 & 0.996 \\
\hline
\end{tabular}




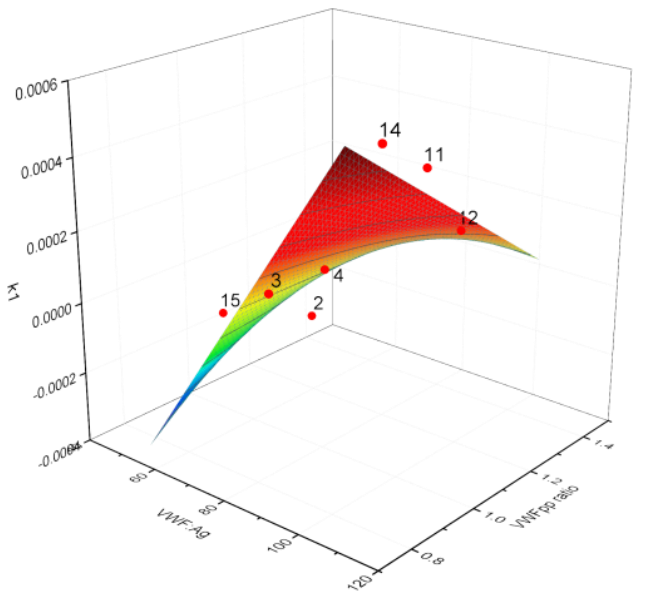

(a)

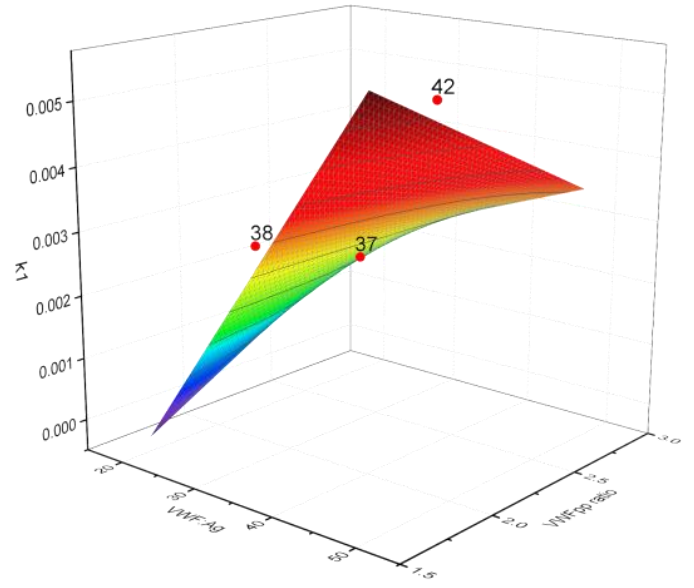

(c)

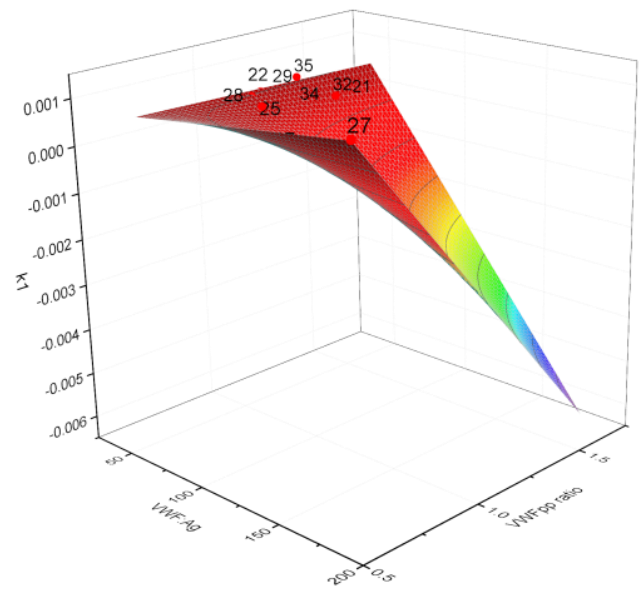

(b)

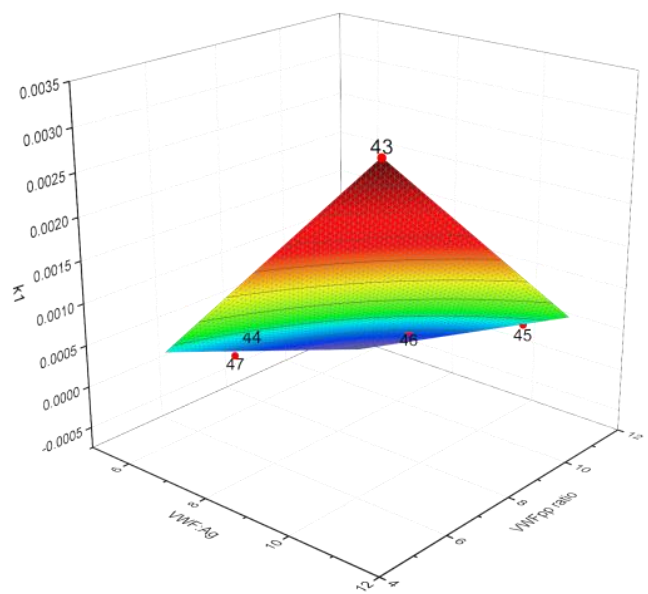

(d)

Figure 5. Linear response model surface with interaction for the proteolytic kinetic constant $k_{1}$ considering subjects HnonO (a), $\mathrm{HO}(b), 2 B(c)$, Vicenza $(d)$.

Table 6. Calculated average $k_{1}$ values and the average estimated $k_{1}$ values.

\begin{tabular}{cccc} 
& $\begin{array}{c}k_{1} \text { calculated by the first level } \\
\text { modified model }\end{array}$ & $\begin{array}{c}k_{1} \text { estimated from the original } \\
\text { model }\end{array}$ & $\begin{array}{c}\text { relative } \\
\text { error }\end{array}$ \\
\cline { 2 - 4 } HnonO & $2.55 \mathrm{E}-04$ & $2.37 \mathrm{E}-04$ & 0.0759 \\
$\mathrm{HO}$ & $4.53 \mathrm{E}-04$ & $6.25 \mathrm{E}-04$ & 0.2752 \\
2B & 0.00484 & 0.00471 & 0.0268 \\
Vicenza & 0.00155 & 0.00149 & 0.0345
\end{tabular}

Equation (5) has then been substituted into the first level of model modification, leading to a second level of model modification of the kinetic model. The second level of model modification led to the new model structure represented in Figure 6, where both the proteolytic kinetic constant $k_{1}$ and the 
elimination kinetic constant $k_{\mathrm{e}}$ are calculated from basal clinical trials data, whereas only the release parameters $k_{0}, D$ and $t_{\max }$ require the estimation from DDAVP data.

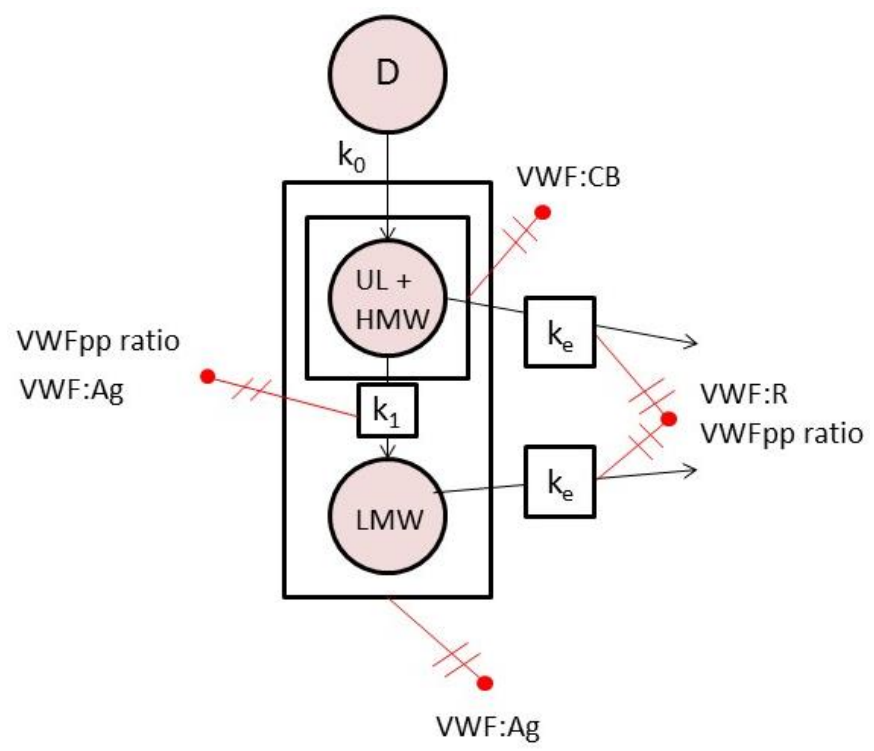

Figure 6. Model scheme obtained after the second level of model modification.

This newly developed model has been validated considering 4 subjects that do not belong to the original pool of subjects used in the development of RSM models, namely patients 1 (HnonO), 19 (HO), 39 (2B) and 50 (Vicenza). Results in terms of predicted VWF:Ag and VWF:CB profiles show an excellent agreement with the simulations obtained from the original model and are reported in Figure 7. 

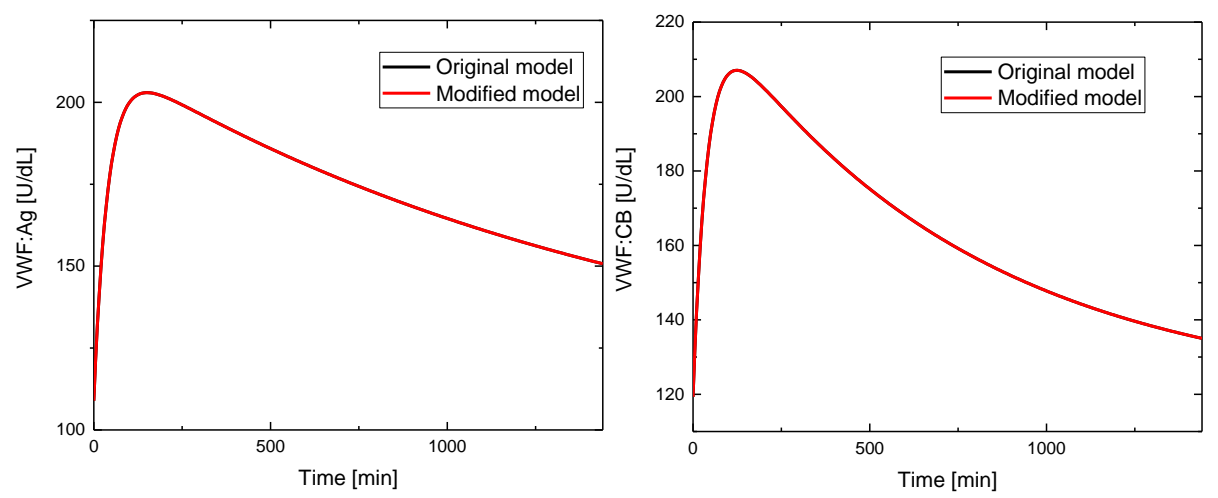

(a)
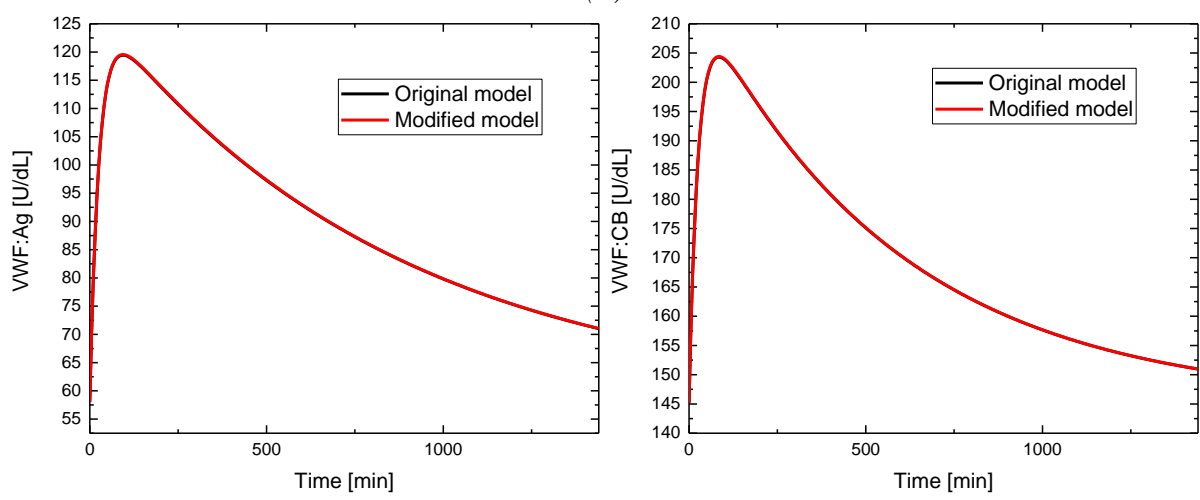

(b)
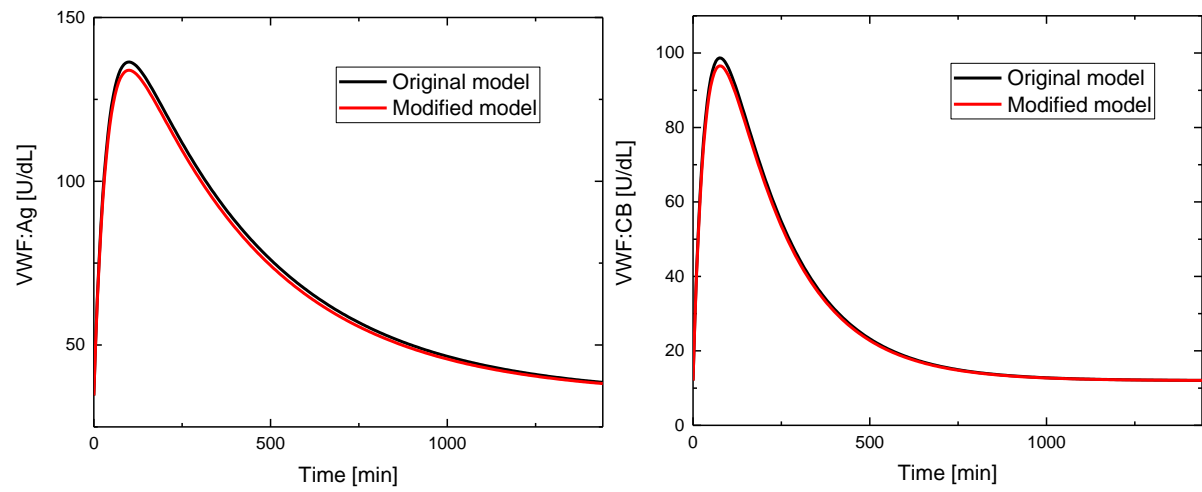

(c)
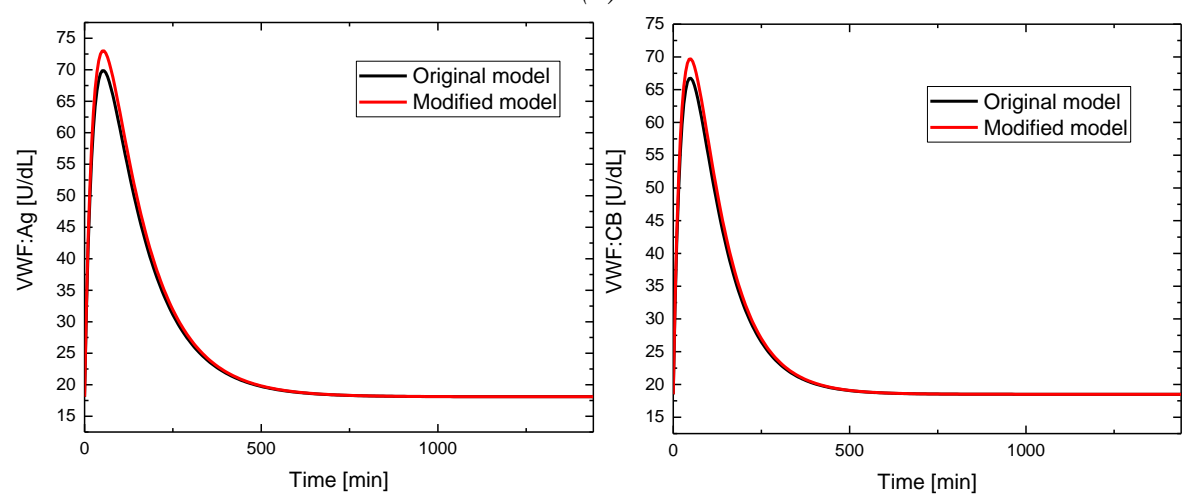

(d)

Figure 7. Comparison between the simulated VWF:Ag (left column) and VWF:CB (right column) profiles obtained with the original model by Ferrari and coworkers [11] (solid black line) and the modified model including basal measurements (red solid line) for (a) patients 1 (HnonO); (b) patient 19 (HO); (c) patient 39 (2B); (d) patient 50 (Vicenza). 
The relative position of response surfaces for each class of subjects is reported in Figure 8. A classification between subject categories can easily be conducted through the newly developed model simply using basal clinical tests data, as the relative position of the response surfaces generated for each VWD category from $k_{1}$ (Figure 8a) and $k_{\mathrm{e}}$ (Figure 8b) correlations do not cross between each other for subjects affected by type 2B and Vicenza VWD. A partial overlap is only observed between healthy $\mathrm{HO}$ and HnonO subjects.

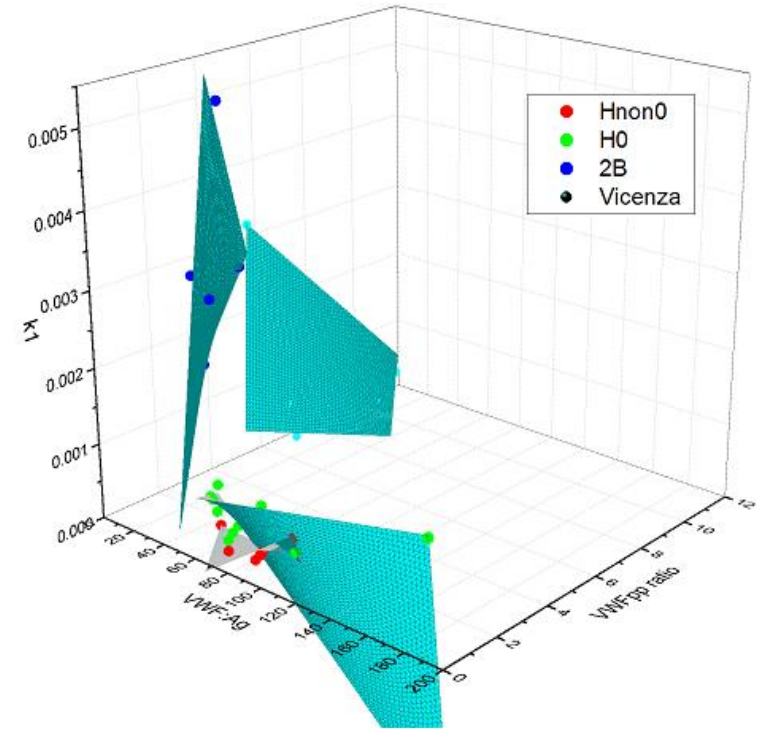

(a)

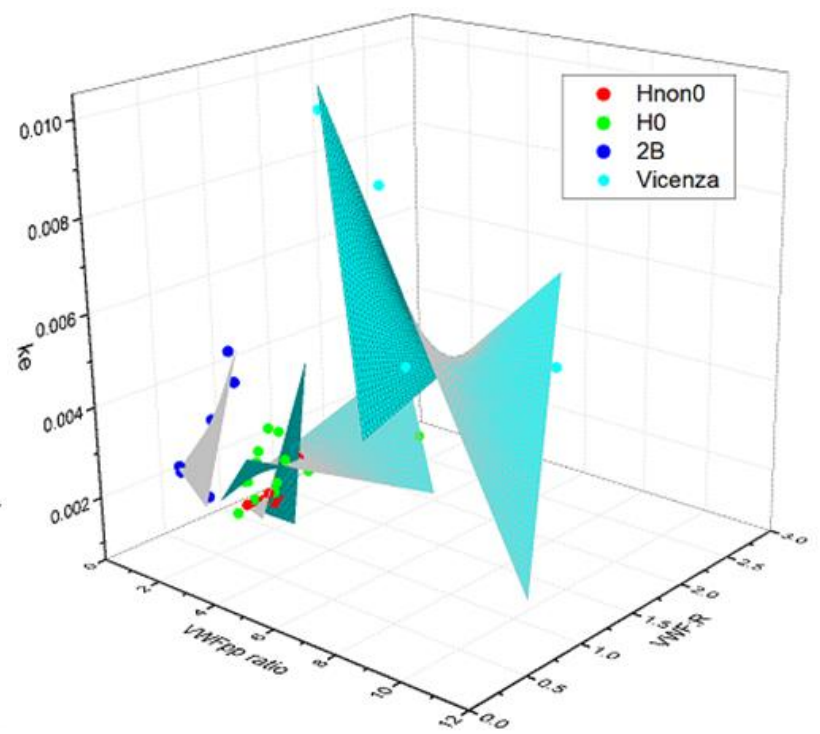

(b)

Figure 8. Relative position of the response surfaces obtained for each class of subjects (healthy O/non-O, $2 B$, Vicenza) using (a) $k_{1}$ and (b) $k_{e}$ correlations.

\subsection{Information analysis}

Information content analysis has been carried out on both the original VWD model [11] and the new modified model of VWD, where correlations (5-6) for $k_{e}$ and $k_{1}$ are included. Results are shown for the original kinetic model (Figure 9a) and the newly developed model (Figure 9b) for four randomly selected patients from each VWD category (patients 11 for $\mathrm{HnonO}, 32$ for $\mathrm{HO}, 37$ for 2B and 45 for Vicenza category). 


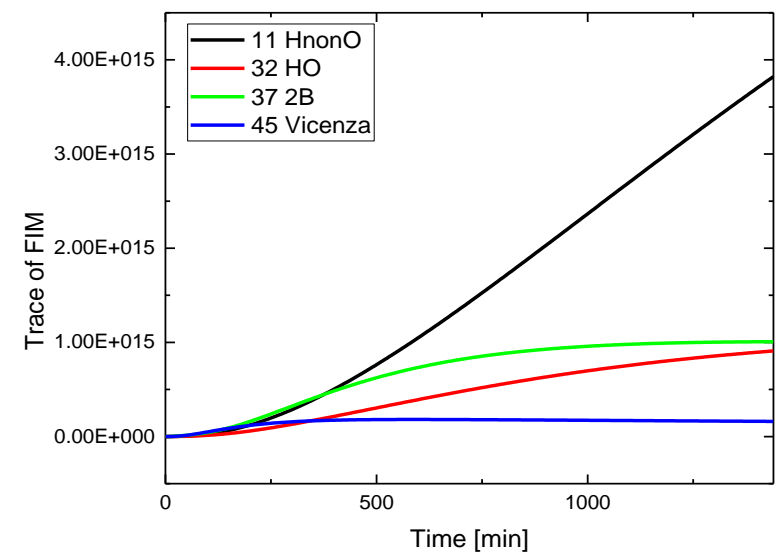

(a)

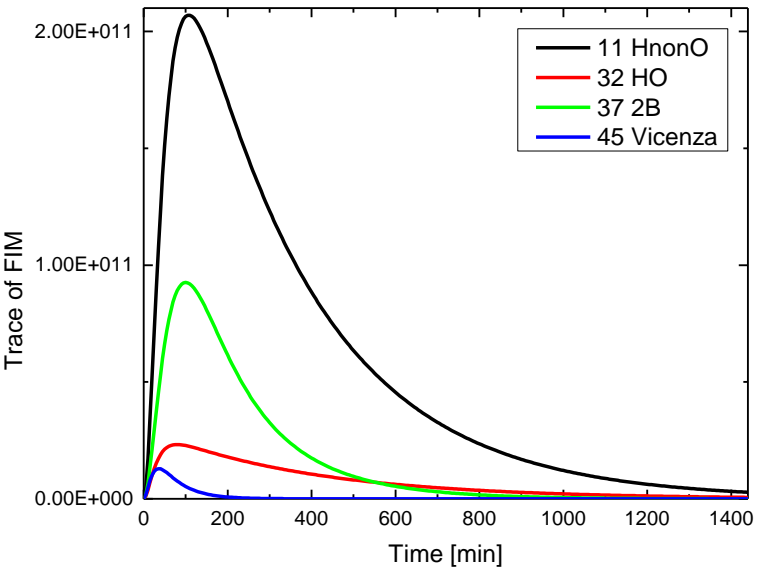

(b)

Figure 9. Trace of FIM for representative subjects from each class (healthy O/non-O, 2B, Vicenza) for (a) the original kinetic model [11] and (b) the modified kinetic model proposed in this study.

As shown in Figure 9a the dynamic profile of the trace of FIM described by (3) and calculated from the original model does not present a maximum during the DDAVP execution time. For this model long execution times are required to gain enough information to estimate the full set of model parameters for each subject. Conversely, the trace peaks at around 3 hours of DDAVP execution considering the newly proposed model (Figure 9b). This means that the amount of information brought by basal clinical data is high enough to drastically reduce the duration of DDAVP test from 24 hours to around 3 hours and redesign a shorter DDAVP test aiming at estimating, for each subject, the release parameters $\left(k_{0}, D\right.$ and $\left.t_{\max }\right)$ only.

\subsection{Optimal redesign of the DDAVP protocol for the estimation of release}

\section{parameters}

The following key activities have been carried out to redesign the DDAVP test [3]:

I) FIM Analysis: The trace of FIM is calculated from (3) for each subject category to evaluate the exact time required by the modified model for reaching the peak in the information content; 
II) Reduction in time of the standard $24 \mathrm{~h}$ DDAVP protocol (with samples at $0,15,30,60,120$, $180,240,480,1440 \mathrm{~min}$ ) considering the time horizon individuated in key activity I. A timereduced DDAVP protocol lasting $3 \mathrm{~h}$ with samples at $0,15,30,60,120,180 \mathrm{~min}$ has been considered as initial guess;

III) Validation of the time-reduced DDAVP protocol proposed in key activity II. A pool of four subjects (one for each category) has been randomly selected at the purpose, generating a random set of experimental data with a normal random error (zero mean) and a standard deviation of $2 \mathrm{U} / \mathrm{dL}$. Parameter estimation has been carried out based on each subject's VWF:Ag and VWF:CB readings using the procedure presented in Supplementary Material 1;

IV) Optimal redesign of the time-reduced DDAVP protocol, where the allocation of sampling points has been optimized for the reduced time-horizon.

In all the simulation activities the software gPROMS $^{\circledR}[20]$ has been used. Key activity IV has been carried out according to the following steps:

- Step 0: selection of reference subjects. Four subjects for each of the considered categories (HnonO, HO, 2B and Vicenza) have been taken as reference to conduct the optimization. The selection of the patients was executed considering subjects with sensibly different values of the model parameters $k_{0}, D$ and $t_{\max }$ even if belonging to the same class. This approach has been chosen to represent the variability of subjects in the pool.

- Step 1: optimization of the sampling time allocation for each subject. Basal clinical data data and PK parameters for each subject (Supplementary Material 2) have been inputted in gPROMS $^{\circledR}$; the time-reduced DDAVP with sampling at $0,15,30,60,120,180 \mathrm{~min}$ has been used as initial guess to optimise the sampling time allocation. Then, the optimization has been carried out comparing three different experimental design criteria: A-optimal, D-optimal and Eoptimal.

- Step 2: definition of the new DDAVP protocol. The DDAVP protocol must be unique for all the categories since, theoretically, the class of belonging of the patients is at the beginning 
unknown. To unify the protocol, the average value of each optimized sampling point for all the selected subjects among the categories has been evaluated.

- Step 3: validation of the new DDAVP protocol. The "worst-case approach" [3] has been applied to test the robustness of the optimized DDAVP designs. The optimized designs have been validated considering critical subjects characterized by the highest determinant of the variance-covariance matrix (i.e. the subjects providing the lowest information for the estimation of kinetic parameters). For these subjects, to evaluate the robustness of the optimized DDAVP designs, in silico experimental data have been generated with zero mean and a standard deviation of $\sigma_{y}=2 \mathrm{U} / \mathrm{dL}$ for both VWF:Ag and VWF:CB. This analysis allows us to understand whether the optimized DDAVP designs are suitable or not to correctly estimate the model parameters.

\subsubsection{Model validation results using a time-reduced DDAVP protocol}

Results from model validation are reported in Figure 10 for a healthy subject (Figure 10a) and a subject affected by VWD type Vicenza (Figure 10b) by simulating the VWF:Ag and VWF:CB responses of the subjects after a time-reduced DDAVP protocol and comparing them with those obtained with the original kinetic model of VWD.

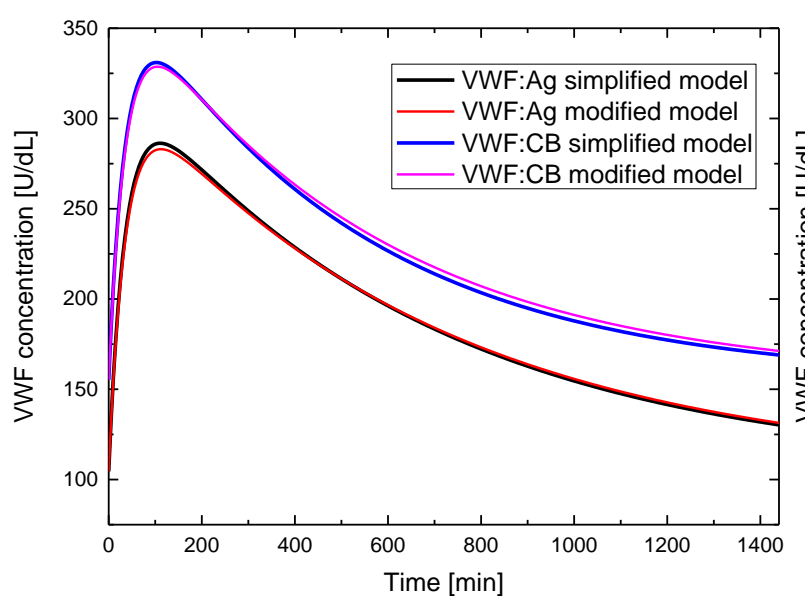

(a)

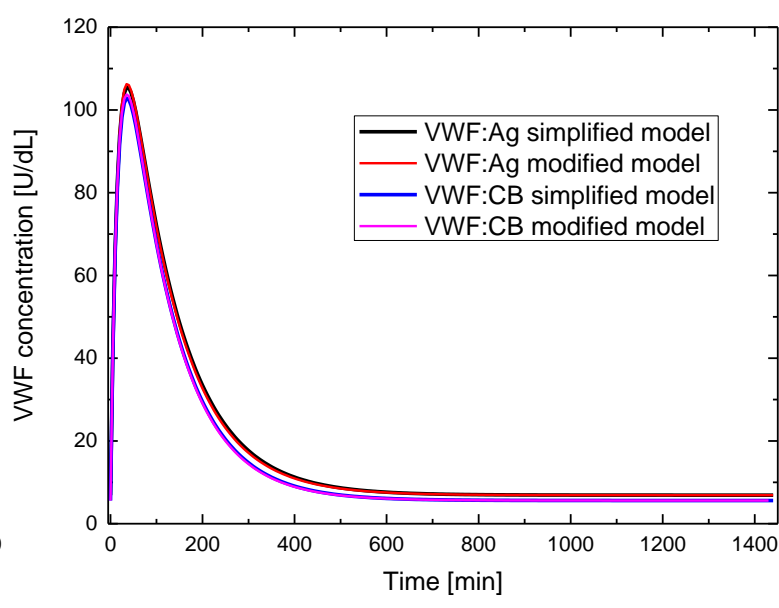

(b)

Figure 10. Comparison between the simulated profiles of VWF:Ag response obtained from the original and the modified kineticmodel for (a) subject 11(healthy non-O) and (b) subject 45 (Vicenza type). 
Results clearly show that the profiles overlap, meaning that the predictive capability of the new kinetic model of VWD is maintained even when a shorter duration for the DDAVP test is used.

\subsubsection{Optimal redesign of the time-reduced DDAVP protocol}

The time-reduced DDAVP protocol has been redesigned considering the pool of subjects reported in Table 7 by comparing three different design criteria: A-, D- and E-optimal. To test the robustness of the optimal designs, four subjects with the highest values of the determinant of the covariance matrix (worst-case approach) have been selected: patients 5 for $\mathrm{HnonO}$ category, 20 for $\mathrm{HO}$ category, 38 for 2B category and 48 for Vicenza category.

Table 7. Reference subjects used for the redesign of the DDAVP test and determinant of the variance-covariance matrix for the different subjects taken as reference. The worst-case scenario subjects used to test the robustness of the optimized DDAVP designs are indicated in boldface.

\begin{tabular}{|c|c|c|c|c|}
\hline & \multicolumn{4}{|c|}{ Reference subjects } \\
\hline HnonO & 11 & 5 & 15 & 14 \\
\hline $\operatorname{Det}\left[V_{\theta}\right]$ & $5.68 \mathrm{E}-03$ & 4.1E-02 & 7.30E.03 & $5.56 \mathrm{E}-03$ \\
\hline HO & 32 & 20 & 30 & 18 \\
\hline $\operatorname{Det}\left[V_{\theta}\right]$ & $5.34 \mathrm{E}-02$ & $5.20 \mathrm{E}-01$ & $3.9 \mathrm{E}-02$ & 7.51E-02 \\
\hline 2B & 37 & 38 & 41 & 40 \\
\hline $\operatorname{Det}\left[V_{\theta}\right]$ & 2.19E-02 & 1.05E-01 & $1.22 \mathrm{E}-02$ & $9.24 \mathrm{E}-03$ \\
\hline Vicenza & 45 & 48 & 43 & 46 \\
\hline $\operatorname{Det}\left[V_{\theta}\right]$ & $1.12 \mathrm{E}-01$ & 2.27 & $3.56 \mathrm{E}-01$ & $5.06 \mathrm{E}-01$ \\
\hline
\end{tabular}

In Table 8, the average values for the optimized DDAVP protocols among all the reference subjects and categories have been reported. A-optimal and E-optimal designs provide the same solutions in terms of allocation of sampling points. Hence, only the following time-reduced DDAVP designs have been tested:

- Time-reduced design;

- A-optimal design;

- D-optimal design.

As shown in Table 8, these three designs are similar in terms of allocation of sampling points. 
Table 8. Time-reduced DDAVP design and average optimized sampling times among the categories using different experimental design criteria.

\begin{tabular}{|c|c|c|c|c|c|c|}
\hline \multicolumn{7}{|c|}{ Experimental Design } \\
\hline Time-reduced DDAVP & \multicolumn{3}{|c|}{ A-optimal design } & \multicolumn{3}{c|}{ D-optimal design } \\
\hline $\begin{array}{c}\text { Sampling times } \\
{[\mathrm{min}]}\end{array}$ & $\begin{array}{c}\text { Average } \\
{[\mathrm{min}]}\end{array}$ & $\begin{array}{c}\text { Rounded } \\
\text { values } \\
{[\mathrm{min}]}\end{array}$ & $\begin{array}{c}\text { Standard deviation } \\
{[\mathrm{min}]}\end{array}$ & $\begin{array}{c}\text { Average } \\
{[\mathrm{min}]}\end{array}$ & $\begin{array}{c}\text { Rounded values } \\
{[\mathrm{min}]}\end{array}$ & $\begin{array}{c}\text { Standard deviation } \\
{[\mathrm{min}]}\end{array}$ \\
\hline 0 & 0 & 0 & 0 & 0 & 0 & 0 \\
\hline 15 & 18.27 & 18 & 6.4 & 20.83 & 20 & 8.0 \\
\hline 30 & 36.51 & 36 & 9.9 & 37.32 & 35 & 9.0 \\
\hline 60 & 61.49 & 60 & 11.6 & 55.39 & 55 & 10.3 \\
\hline 120 & 156.84 & 155 & 42.9 & 141.09 & 140 & 44.5 \\
\hline 180 & 171.83 & 172 & 42.9 & 165.98 & 166 & 46.5 \\
\hline
\end{tabular}

The robustness of these protocols has been analysed through a "worst-case" approach. All the designs have been tested and validated generating a set of experimental data in silico for the most critical patient (i.e. the patient in each category of VWD characterised by a higher expected uncertainty on model parameters) and estimating the release parameters $k_{0}, D$ and $t_{\max }$. As reported in Table 9 and 10, both the D- and A- optimal designs allow estimating $k_{0}, D$ and $t_{\text {max }}$ with a similar precision (t-values and confidence intervals) but the D-optimal design provides a better accuracy in the estimation of the release parameters of patient 38 (i.e. estimated values are closer to the ones obtained from a 24 hours long DDAVP protocol). Particularly $D$ and $k_{0}$, which are the parameters defining the amount of VWF released, are estimated with a higher precision when a D-optimal protocol is used on this patient, a subject affected by $2 \mathrm{~B}$ type VWD who appears to be the most critical subject to characterise in terms of kineticparameters. Both the A-optimal and D-optimal designs allow an accurate calculation of the release parameters for the other subjects. 
Table 9. Parameter estimation results obtained from the A-optimal protocol in terms of estimated values and statistics including 95\% confidence intervals, $t$-values and standard deviation. Parameters showing $a$ relative deviation greater than 10\% from the $24 \mathrm{~h}$ long DDAVP estimated values are reported in boldface.

\begin{tabular}{|c|c|c|c|c|c|c|}
\hline \multirow[b]{3}{*}{$\mathrm{D}$} & \multirow[b]{3}{*}[\mathrm{U}]{} & \multicolumn{5}{|c|}{ Patient 5} \\
\hline & & 24 h DDAVP & $\begin{array}{c}\text { A-optimal } \\
\text { design }\end{array}$ & $\begin{array}{l}95 \% \text { conf. } \\
\text { Interval }\end{array}$ & 95\% t-value & "Standard deviation \\
\hline & & 407.020 & $405.240(-0.5 \%)$ & 1300.000 & 0.320 & 570.000 \\
\hline $\mathrm{k}_{0}$ & {$\left[\mathrm{~min}^{-1}\right]$} & 0.029 & $0.029(+0 \%)$ & 0.041 & 0.710 & 0.018 \\
\hline \multirow[t]{3}{*}{$t_{\max }$} & {$[\mathrm{min}]$} & 69.190 & $69.190(+0 \%)$ & 11.000 & 6.501 & 4.800 \\
\hline & & \multicolumn{5}{|c|}{ Patient 20 } \\
\hline & & 24 h DDAVP & $\begin{array}{c}\text { A-optimal } \\
\text { design }\end{array}$ & $\begin{array}{l}95 \% \text { conf. } \\
\text { Interval }\end{array}$ & $95 \%$ t-value & Standard deviation \\
\hline $\mathrm{D}$ & {$[\mathrm{U}]$} & 412.170 & $416.930(+1.1 \%)$ & 1900.000 & 0.210 & 870.000 \\
\hline $\mathrm{k}_{0}$ & {$\left[\mathrm{~min}^{-1}\right]$} & 0.031 & $0.032(+3.2 \%)$ & 0.096 & 0.340 & 0.043 \\
\hline \multirow[t]{3}{*}{$t_{\max }$} & {$[\mathrm{min}]$} & 40.090 & $41.920(+4.5 \%)$ & 28.000 & 1.500 & 13.000 \\
\hline & & \multicolumn{5}{|c|}{ Patient 38} \\
\hline & & 24 h DDAVP & $\begin{array}{c}\text { A-optimal } \\
\text { design }\end{array}$ & $\begin{array}{l}\text { 95\% conf. } \\
\text { Interval }\end{array}$ & $95 \%$ t-value & "Standard deviation \\
\hline $\mathrm{D}$ & {$[\mathrm{U}]$} & 930.250 & $467.170(-49.8 \%)$ & 1500.000 & 0.310 & 680.000 \\
\hline $\mathrm{k}_{0}$ & {$\left[\mathrm{~min}^{-1}\right]$} & 0.008 & $0.018(+125.2 \%)$ & 0.025 & 0.740 & 0.011 \\
\hline \multirow[t]{3}{*}{$t_{\max }$} & {$[\mathrm{min}]$} & 176.320 & $109.200(-37.9 \%)$ & 20.000 & 5.400 & 9.200 \\
\hline & & \multicolumn{5}{|c|}{ Patient 48} \\
\hline & & 24 h DDAVP & $\begin{array}{c}\text { A-optimal } \\
\text { design }\end{array}$ & $\begin{array}{l}\text { 95\% conf. } \\
\text { Interval }\end{array}$ & $95 \%$ t-value & Standard deviation \\
\hline $\mathrm{D}$ & {$[\mathrm{U}]$} & 50.600 & 47.800 & 1000.000 & 0.046 & 460.000 \\
\hline $\mathrm{k}_{0}$ & {$\left[\mathrm{~min}^{-1}\right]$} & 0.099 & $0.099(+0 \%)$ & 0.610 & 0.160 & 0.270 \\
\hline$t_{\max }$ & {$[\mathrm{min}]$} & 34.540 & $34.540(+0 \%)$ & 9.900 & 3.500 & 4.500 \\
\hline
\end{tabular}

Table 10. Parameter estimation results obtained from the D-optimal protocol in terms of estimated values and statistics including 95\% confidence intervals, $t$-values and standard deviation. Parameters showing a relative deviation greater than $10 \%$ from the $24 \mathrm{~h}$ long DDAVP estimated values are reported in boldface.

\begin{tabular}{|c|c|c|c|c|c|c|}
\hline \multirow[b]{3}{*}{$\mathrm{D}$} & \multirow[b]{3}{*}[\mathrm{U}]{} & \multicolumn{5}{|c|}{ Patient 5} \\
\hline & & 24 h DDAVP & $\begin{array}{c}\text { D-optimal } \\
\text { design }\end{array}$ & $\begin{array}{l}95 \% \text { conf. } \\
\text { Interval }\end{array}$ & $95 \%$ t-value & Standard deviation \\
\hline & & 407.015 & $415.830(+2 \%)$ & 1200.000 & 0.350 & 540.000 \\
\hline $\mathrm{k}_{0}$ & {$\left[\mathrm{~min}^{-1}\right]$} & 0.029 & $0.030(+3.4 \%)$ & 0.037 & 0.820 & 0.017 \\
\hline \multirow[t]{3}{*}{$t_{\max }$} & {$[\mathrm{min}]$} & 69.190 & $73.400(+6.0 \%)$ & 9.300 & 7.900 & 4.200 \\
\hline & & \multicolumn{5}{|c|}{ Patient 20} \\
\hline & & 24 h DDAVP & $\begin{array}{c}\text { D-optimal } \\
\text { design }\end{array}$ & $\begin{array}{l}\text { 95\% conf. } \\
\text { Interval }\end{array}$ & $95 \%$ t-value & Standard deviation \\
\hline $\mathrm{D}$ & {$[\mathrm{U}]$} & 412.170 & $419.700(+1.8 \%)$ & 1900.000 & 0.220 & 860.000 \\
\hline $\mathrm{k}_{0}$ & {$\left[\mathrm{~min}^{-1}\right]$} & 0.031 & $0.032(+3.2 \%)$ & 0.093 & 0.350 & 0.042 \\
\hline \multirow[t]{3}{*}{$\mathrm{t}_{\max }$} & {$[\mathrm{min}]$} & 40.090 & $43.150(+7.6 \%)$ & 26.000 & 1.700 & 12.000 \\
\hline & & \multicolumn{5}{|c|}{ Patient 38} \\
\hline & & 24 h DDAVP & $\begin{array}{c}\text { D-optimal } \\
\text { design }\end{array}$ & $\begin{array}{l}95 \% \text { conf. } \\
\text { Interval }\end{array}$ & $95 \%$ t-value & Standard deviation \\
\hline $\mathrm{D}$ & {$[\mathrm{U}]$} & 930.250 & $946.190(+1.7 \%)$ & 3100.000 & 0.300 & 1400.000 \\
\hline $\mathrm{k}_{0}$ & {$\left[\mathrm{~min}^{-1}\right]$} & 0.008 & $0.012(+\mathbf{5 0 \%})$ & 0.023 & 0.560 & 0.010 \\
\hline \multirow[t]{3}{*}{$\mathrm{t}_{\max }$} & {$[\mathrm{min}]$} & 176.320 & $104.470(+\mathbf{4 0 \%})$ & 22.000 & 4.700 & 10.000 \\
\hline & & \multicolumn{5}{|c|}{ Patient 48} \\
\hline & & 24 h DDAVP & $\begin{array}{c}\text { D-optimal } \\
\text { design }\end{array}$ & $\begin{array}{l}95 \% \text { conf. } \\
\text { Interval }\end{array}$ & $95 \%$ t-value & Standard deviation \\
\hline $\mathrm{D}$ & {$[\mathrm{U}]$} & 50.600 & $50.570(+0 \%)$ & 1200.000 & 0.043 & 530.000 \\
\hline $\mathrm{k}_{0}$ & {$\left[\mathrm{~min}^{-1}\right]$} & 0.099 & $0.098(-1.0 \%)$ & 0.650 & 0.150 & 0.290 \\
\hline$t_{\max }$ & {$[\mathrm{min}]$} & 34.540 & $34.540(+0 \%)$ & 9.700 & 3.600 & 4.400 \\
\hline
\end{tabular}


Figure 11 shows how both the A-optimal and D-optimal designs are able to guarantee a good fitting of the experimental data for one of the critical patients considered in the study (patient 5). Analogous results have been obtained for the other critical subjects, and have not been reported for the sake of conciseness.
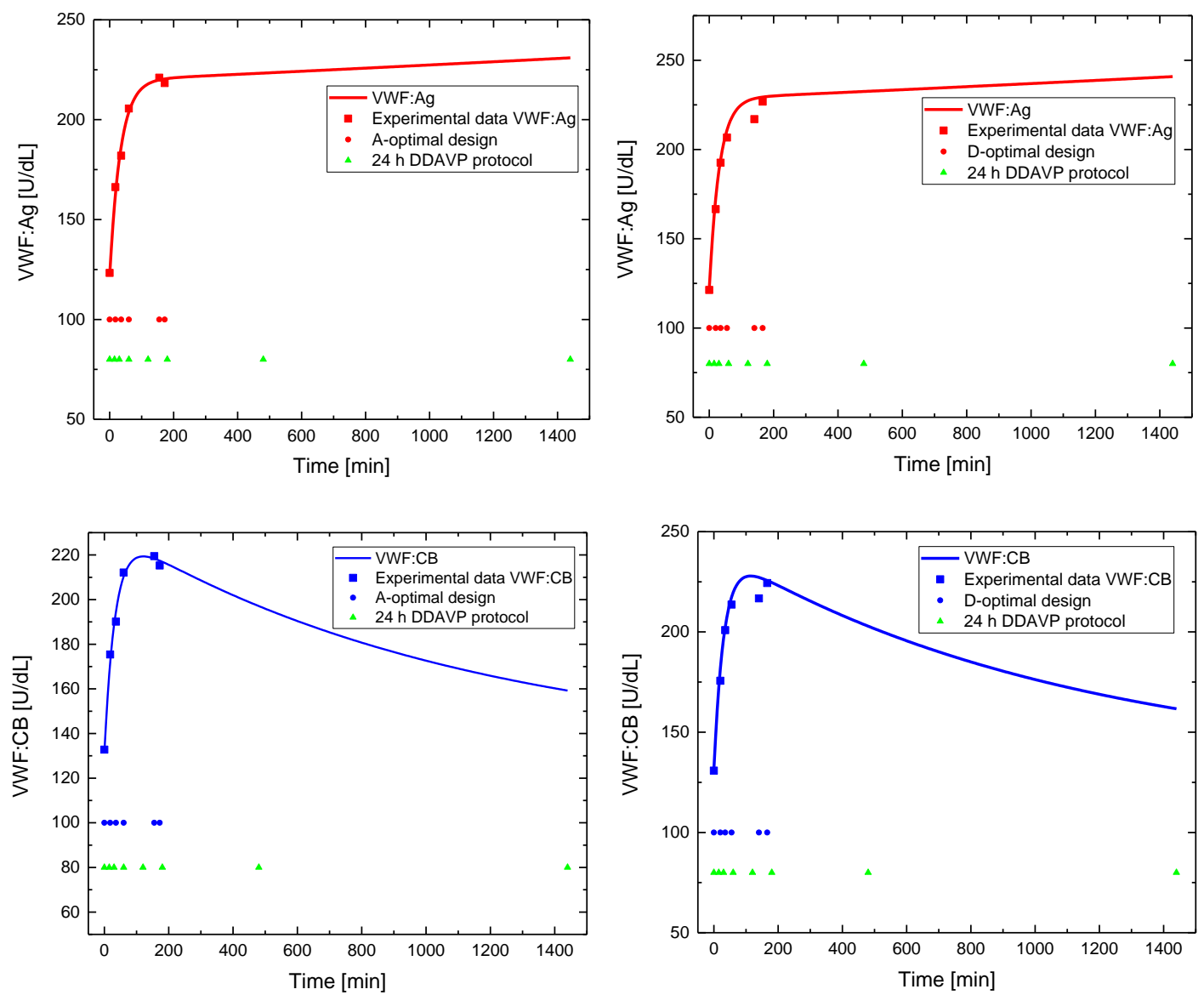

Figure 11. VWF:Ag and VWF:CB profiles for patient 5 using the A-optimal (left) and D-optimal (right) designs. Blue squares represent VWF measurements, blue diamonds the allocation of sampling points according to an A-optimal MBDoE and the green triangles the allocation of sampling points in a standard 24 h DDAVP.

\subsection{Practical implications of the proposed optimal sampling scheduling}

In the everyday clinical procedures, it is practically impossible to sample with a resolution in time lower than 10 minutes. Therefore, referring to the DDAVP execution, taking a final sample at 172 minutes (A-optimal design) or at 180 min (time-reduced design) does not make a substantial difference because an error of \pm 8 minutes in the sampling procedure can be admitted. A $3 \mathrm{~h}$ long time-reduced DDAVP would require sampling at $0,15,30,60,120,180 \mathrm{~min}$. This test is very 
simple, as it is only a reduction in time of the standard 24 hours long DDAVP and would not have any impact on the current clinical procedure. A D-optimal would require a sampling at $0,20,35,55$, 140, $166 \mathrm{~min}$. The last two samples are closer in time but still manageable from the point of view of the clinical test, and these last samples have an impact on the accuracy of the estimates, as clearly shown in the previous section.

\section{Conclusions}

The kinetic model proposed in [11] is efficient to quantify the mechanisms of VWF release, proteolysis and elimination, but requires a 24 hours long complex test (DDAVP) to be executed to achieve a statistically satisfactory estimation of the metabolic parameters for each subject.

In this work, a procedure has been proposed for model identification exploiting the complementary information derived by basal clinical trials: VWFpp, to quantify VWF elimination from the bloodstream; VWF:Ag, to evaluate the concentration of VWF molecule in the bloodstream; VWF:CB, to analyse the VWF ability to bind collagen. From them, two other key physiological quantities are derived: VWFpp ratio and VWF:CB ratio. RSM has been applied for the development of correlations explicitly relating the kinetic parameters $k_{1}$ and $k_{\mathrm{e}}$ of the original kinetic model of VWD to the data obtained from basal clinical trials. In particular, $k_{1}$ is derived by VWF:Ag and VWFpp ratio basal tests, whereas $k_{\mathrm{e}}$ is calculated from VWFpp ratio and VWF:CB ratio measurements. These correlations have been used in a new modified kinetic model where information from basal clinical tests is exploited. $\mathrm{MBDoE}$ techniques have been used to design a shorter DDAVP test for the estimation of the release parameters only $\left(k_{0}, D\right.$ and $\left.t_{\text {max. }}\right)$ and to optimally allocate the sampling points in a DDAVP test where the duration has been drastically reduced from 24 hours (standard DDAVP) to three hours. The new model has been tested in silico on subjects that do not belong to the original validation pool. Results demonstrate that the profiles generated with the modified model and the original model overlap, thus meaning that the predictive capability of the original model has been maintained. The reduction of DDAVP duration is a 
remarkable achievement because it allows patients to undergo a less stressful clinical procedure and it facilitates clinical management in terms of both economical and organizational aspects. Future work will be carried out to extend the model validity to different VWD types and to reinforce model validation by applying the time-reduced DDAVP to characterize the VWF:Ag and VWF:CB profiles of new critical patients which do not belong to the pool of subjects already considered.

\section{References}

[1] H. Akaike, A new look at the statistical model identification, IEEE Trans. Automat. Contr. 19 (1974) 716-723.

[2] M.K. Al-Banna, A.W. Kelman, B. Whiting, Experimental design and efficient parameter estimation in population pharmacokinetics, J. Pharmacokinet. Biopharm. 18 (1990) 347-358.

[3] S.P. Asprey, S. Macchietto, Designing robust optimal dynamic experiments. J. Process Control 12 (2002) 545-556.

[4] Y. Bard, Nonlinear Parameter Estimation, Academic Press, New York, 1974.

[5] G. Box, D. Benhken, Some new 3 level designs for the study of quantitative variables, Technometrics 2 (1960) 455-475.

[6] A. Casonato , V. Daidone, R. Padrini, Assessment of von Willebrand factor propeptide improves the diagnosis of von Willebrand disease, Semin. Thromb. Hemost. 37 (2011) 456463.

[7] A. Casonato, E. Pontara, F. Sartorello, Identifying type Vicenza von Willebrand disease. J Lab Clin. Med. 147 (2006) 96-102.

[8] C. Castaldello, F. Galvanin, A. Casonato, R. Padrini, M. Barolo, F. Bezzo, A Model-Based Protocol for the Diagnosis of von Willebrand Disease, Can. J. Chem. Eng. 96 (2018) 628-638.

[9] S. C. L. M. Cremers, E. M. Scholten, R. C. Schoemaker, E. G. W. M. Lentjes, P. Vermeij, L. C. Paul, J. den Hartigh, J. W. de Fijter, A compartmental pharmacokinetic model of cyclosporin and its predictive performance after Bayesian estimation in kidney and 
simultaneous pancreas-kidney transplant recipients, Nephr. Dial. Transplant. 18 (2003) 12011208.

[10] V. Fedorov, S. Leonov, Optimal design for nonlinear response models, Taylor \& Francis, Boca Raton, 2014.

[11] M. Ferrari, , F. Galvanin, M. Barolo, V. Daidone, R. Padrini, F. Bezzo, A. Casonato, A Mechanistic Model to Quantify von Willebrand Factor Release, Survival and Proteolysis in Patients with von Willebrand Disease, Thromb. Haemost. 118 (2018) 309-319.

[12] F. Galvanin, C. Ballan, M. Barolo, F. Bezzo, A general model-based design of experiments approach to achieve practical identifiability of pharmacokinetic and pharmacodynamic models, J Pharmacokinet. Pharmacodyn. 40 (2013) 451-467.

[13] F. Galvanin, M. Barolo, R. Padrini, A. Casonato, F. Bezzo, A model-based approach to the automatic diagnosis of von Willebrand disease, AIChE J. 60 (2014) 1718-1727.

[14] E. Groot, R. Fijnheer, S. Sebastian, The active conformation of von Willebrand factor in patients with thrombotic thrombocytopenic purpura in remission, J. Thromb. Haemost. 7 (2009) 962-969.

[15] J. M. Laínez-Aguirre, L. Mockus, S. Orçun, G. Blau, G. V. Reklaitis, A Decomposition Strategy for the Variational Inference of Complex Systems, Technometrics 58 (2016) 84-94.

[16] P. J. Lenting, C. J. M. Van Schooten, C. V. Denis. Clearance mechanisms of von Willebrand factor and factor VIII, J. Thromb. Haemost. 5 (2007) 1353-1360.

[17] D. Lillicrap, Von Willebrand disease-phenotype versus genotype: Deficiency versus disease, Thromb. Res. 87 (2007) 57-64.

[18] D. Menache, D. L. Aronson, F. Darr, R. R. Montgomery, J. C. Gill, C. M. Kessler, J. M. Lusher, P. D. Phatak, A. D. Shapiro, A. R. Thompson, G. C White, Pharmacokinetics of von Willebrand factor and factor VIIIC in patients with severe von Willebrand disease (type 3 VWD): estimation of the rate of factor VIIIC synthesis, Br. J. Haematol. 94 (1996) 740-745.

[19] OriginLab Corporation, Origin 8 User Guide, Northampton, 2007. 
[20] Process Systems Enterprise, gPROMS Advanced User Guide, Process Systems Enterprise Ltd., London, 2004.

[21] J. E. Sadler, Von Willebrand disease type 1: a diagnosis in search of a disease, Blood 101 (2003) 2089-2093.

[22] J. D. Sweeney, L.A. Hoerning, Intraplatelet von Willebrand factor and AB0 blood group, Thromb. Res. 68 (1992) 393-398.

[23] V. H. Tam, S. L. Preston, G. L. Drusano. Optimal sampling schedule design for populations of patients. Antimicrob. Agents Chemother. 47 (2003) 2888-2891.

[24] B. Zieger, U. Budde, U. Jessat, R. Zimmermann, M. Simon, R. Katzel, A.H. Sutor, New Families with von Willebrand disease type 2M (Vicenza), Thromb. Res. 87 (1997) 57-64. 